\title{
Probing stress and fracture mechanism in encapsulated thin silicon solar cells by synchrotron X-Ray microdiffraction
}

\author{
V.A. Handara $^{1,2}$, I. Radchenko ${ }^{1}$, S.K. Tippabhotla ${ }^{1}$, Karthic. R.Narayanan ${ }^{1}$, \\ G. Illya $^{3}$, M. Kunz ${ }^{4}$, N. Tamura ${ }^{4}$, A. S. Budiman ${ }^{1, a^{*}}$ \\ ${ }^{1}$ Singapore University of Technology and Design, Singapore 487372, \\ ${ }^{\mathrm{a}}$ Formerly of SunPower Corporation, R\&D, San Jose, CA 95134. \\ ${ }^{2}$ Center for Solar Photovoltaics Materials \& Technology (CPV), \\ Surya University, Tangerang, Indonesia 15810. \\ ${ }^{3}$ Buddhi Dharma University, \\ Tangerang, Indonesia, 15115. \\ ${ }^{4}$ Advanced Light Source (ALS), \\ Lawrence Berkeley National Laboratory (LBNL), Berkeley, CA 94720. \\ *suriadi@alumni.stanford.edu
}

\begin{abstract}
$\underline{\text { Abstract }}$
Thin $(<150 \mu \mathrm{m})$ silicon solar cell technology is attractive due to the significant cost reduction associated with it. Consequently, fracture mechanisms in the thin silicon solar cells during soldering and lamination need to be fully understood quantitatively in order to enable photovoltaics (PV) systems implementation in both manufacturing and field operations. Synchrotron X-ray Microdiffraction ( $\mu$ SXRD) has proven to be a very effective means to quantitatively probe the mechanical stress which is the driving force of the fracture mechanisms (initiation, propagation, and propensity) in the thin silicon solar cells, especially when they are already encapsulated. In this article, we present the first ever stress examination in encapsulated thin silicon solar cells and show how nominally the same silicon solar cells encapsulated by different polymer encapsulants could have very
\end{abstract}


different residual stresses after the lamination process. It is then not difficult to see how the earlier observation, as reported by M. Sander et al [1], of very different fracture rates within the same silicon solar cells encapsulated by different Ethylene Vinyl Acetate (EVA) materials could come about. The complete second degree tensor components of the residual stress of the silicon solar cells after lamination process are also reported in this paper signifying the full and unique capabilities of the Synchrotron X-Ray Microdiffraction technique not only for measuring residual stress but also for measuring other potential mechanical damage within thin silicon solar cells.

\section{Introduction}

Silicon materials especially monocrystalline silicon have been used extensively in the photovoltaics (PV) industry due to high efficiency capability $(\sim 21 \%$ conversion efficiency). Recently, there has been a progress in reducing monocrystalline silicon wafer thickness below $150 \mu \mathrm{m}$ due to significant cost reduction associated with it while maintaining the same reliability and durability $[1,2]$. In the northern hemisphere countries where majority of PV markets exist, some extreme climatic conditions (i.e. extreme temperature, snow, wind and thermal cycles) exist and they have major degrading impacts on PV module reliability in terms of higher stress within the silicon solar cells. These external/environmental conditions increase the built-in stress, in addition to the inherent residual stresses developed during the manufacturing processes such as lamination and soldering. Consequently, extreme climatic conditions lead to the increase of fracture propensity within the solar cells reducing PV module's reliability and efficiency significantly [3-5].

To address this issue, solar PV module lamination materials and processes need to be fully understood $[6,7]$. The encapsulant materials, most commonly called as ethylene vinyl acetate (EVA) used for solar PV lamination must fulfill specific criteria such as high optical transmission, good dielectric properties, strong mechanical compliance and high adhesion strength. However, the sole effect of encapsulant materials on the silicon solar cell stresses (i.e. the residual and deviatoric stresses) and the ensuing fracture events have not been further explored in literature [8, 9]. In the recent report by Dietrich et al. [6], it was clearly shown that higher stiffness encapsulant induces cell cracks on PV module at lower 
load and vice versa for lower stiffness encapsulants. The effect of encapsulation stiffness at cold environment conditions on fracture propensity within PV module was reported by Mickiewicz et al. [10]. This preliminary evidence regarding the effect of laminating materials and processes motivated us to understand more into the fundamental fracture mechanisms. Thus, further research into quantitative investigation of the driving force of the fracture events, ie. the mechanical stress, will be investigated in this paper.

Characterizing stress quantitatively on silicon-based PV module which has been laminated by $\sim 0.5 \mathrm{~mm}$ transparent polymer encapsulants on both sides of the module requires a synchrotron X-ray technique [8-9,11]. Synchrotron X-ray Microdiffraction ( $\mu \mathrm{SXRD})$ is a unique technique allowing high resolution, non-destructive quantitative stress and microstructure evolution examination of crystalline materials which has been used effectively and more recently in many advanced systems and novel technologies, such as in micro/nano-electronics [112-15] and nanomaterials/technologies [16 - 20].

In this article, this technique will again be utilized to unravel important insights about deformation mechanisms leading to high stress and thus fracture propensity in encapsulated silicon solar cells as basic components of PV modules. The corresponding Xray beam is capable of reaching the silicon solar cell material (and returning to the X-ray detector/camera after being scattered by the solar cell) even though it is buried under $\sim 0.5$ $\mathrm{mm}$ encapsulation polymer (please refer to Appendix-A for X-ray penetration depth in different materials). This is a unique capability as it would allow stress and microstructure evolution examination within silicon solar cells for instance during high temperature, high pressure lamination process, or even during the operation (in operando) of the PV systems involving thermal cycle or mechanical loading. Also, this kind of measurement is not hitherto possible using charged particle microscopy technique such as Scanning Electron Microscopy (SEM) and Transmission Electron Microscopy (TEM) or surface sensitive techniques such as micro-Raman spectroscopy. This technique could further lead to unravelling of potentially important and critical insights about what is really happening in the silicon solar cell during lamination or what the deformation mechanisms are in the silicon solar cells during thermal cycling or mechanical loading.. Our recent study on laminated solar cells using this technique at the Advanced Light Source (ALS), Berkeley Lab has thus garnered much attention especially from the industry as illustrated in the 
Berkeley science highlight [21] of our work in 2013. Further information regarding encapsulated silicon solar cell characterization using this technique has been successfully published by our group [8]. Therefore in this paper, the authors would like to demonstrate even further the capability of the $\mu$ SXRD technique to predict residual stress (and thus fracture origins) within silicon solar cells laminated by different encapsulation materials.

\section{Experimental Setup}

A series of PV mini modules/laminates (single-cell coupons with commercially available monocrystalline silicon solar cells) were prepared using the two different encapsulants: an industry standard EVA (labelled as "Encapsulant A") and a commercially available thermoplastic (labelled simply as "Encapsulant B" due to proprietary reason). Each of laminated mini module consists of a glass, a front encapsulant layer, a $125 \mathrm{~mm} \mathrm{x}$ $125 \mathrm{~mm}$ SunPower interdigitated back contact (IBC) solar cell (SunPower Corporation, San Jose, CA) made of monocrystalline silicon wafer and a back encapsulant layer. The two samples (i.e. Sample A, with the Encapsulant A, and Sample B, with the Encapsulant B) are otherwise nominally the same samples with the same silicon solar cells and solder joint materials and processing (using high volume, highly automated stringing process), except for the encapsulant materials. However, different encapsulant materials (especially one being a thermoset while the other being a thermoplastic), yields some inevitable difference in the lamination process parameters/recipes, although the maximum lamination temperatures both samples experienced in the present were intentionally fixed at the same temperature. Residual stress measurements in the silicon cell around the solder joint (Figure 1a) were performed by Synchrotron X-ray Microdiffraction ( $\mu$ SXRD) at the Beamline 12.3.2, Advanced Light Source (ALS) of the Lawrence Berkeley National Laboratory [22-24]. The main reason to use mini (single-cell) module is based on the size of X-ray apparatus stage holder. 
(a)

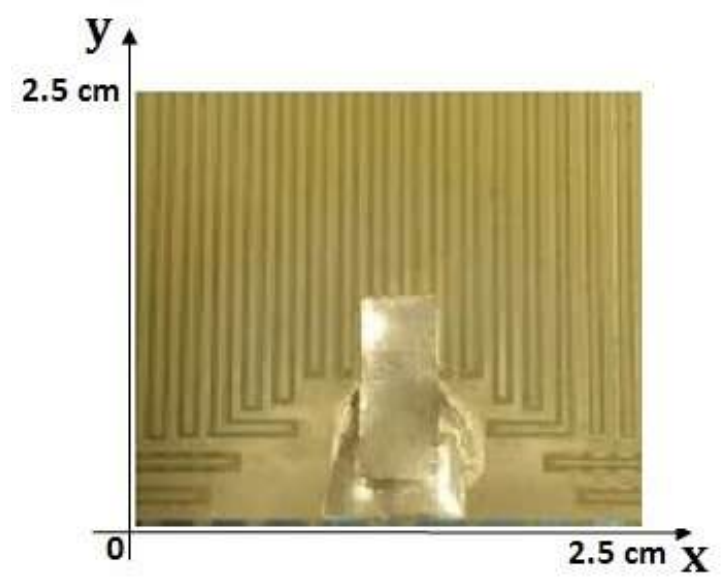

(b)

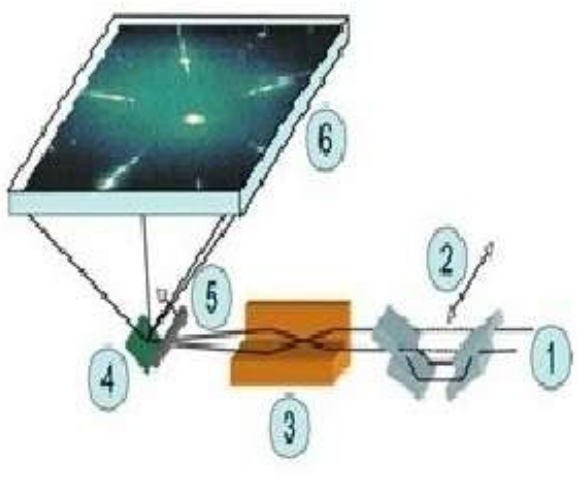

Figure 1: (a) Image of encapsulated Sunpower IBC silicon solar cell around solder joint and (b) Schematic layout of the synchrotron X-Ray microdiffraction ( $\mu$ SXRD) beamline at the Beamline 12.3.2 in the Advanced Light Source, Berkeley Lab [22]

\subsection{Synchrotron X-ray microdiffraction ( $\mu$ SXRD) experiment}

The schematic of the beamline setup was shown in Figure 1b. The polychromatic Xray beam, which is generated by a superconducting magnet source is re-focused at the entrance of the experimental hutch (1) by a $700 \mathrm{~mm}$ platinum-coated silicon toroidal mirror operating at a grazing angle of $4.5 \mathrm{mrad}$ (2). Final focusing is performed by Kirkpatrick - Baez (KB) mirrors (3) consisting of an orthogonal pair of $100 \mathrm{~mm}$ long tungsten coated silicon substrate bent to an elliptical shape. The mini PV module is mounted on a $X Y$ high precision stage having range of $10 \mathrm{~cm}(4)$. As the beam strikes the mini module, it will be diffracted into multiple beam patterns (5), which are then captured by Charge Coupled Device (CCD) detector placed at a distance of $140 \mathrm{~mm}$ above the module sample (6). The CCD detector (having the resolution of $0.03^{\circ}$ and active area of $179 \times 169$ $\mathrm{mm}^{2}$ ) is comprised of pixels in which the relative change in position and size of the peak are extracted [24].

The X-ray penetration depth in a sample depends on the X-ray energy, the incidence angle and the material parameters of the sample (chemical formula and density). X-ray beam penetration depths (for a $45^{0}$ incidence angle and energies, 5-25 keV) for different 
materials of the subject PV module samples were plotted in Appendix-A. Unlike standard X-ray diffraction and Raman spectroscopy, X-ray microdiffraction offers unique capability by which the high energy polychromatic X-ray beam is capable of penetrating encapsulant layer allowing quantitative stress measurement within laminated silicon solar cells.

(a)

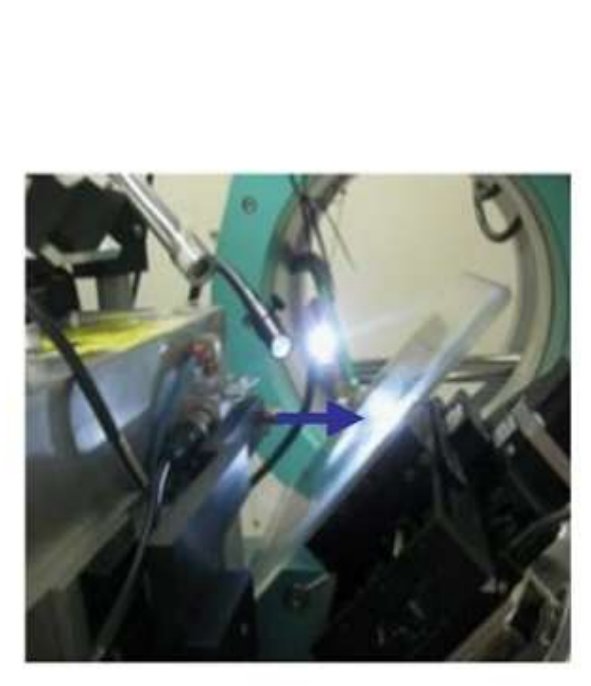

(b)

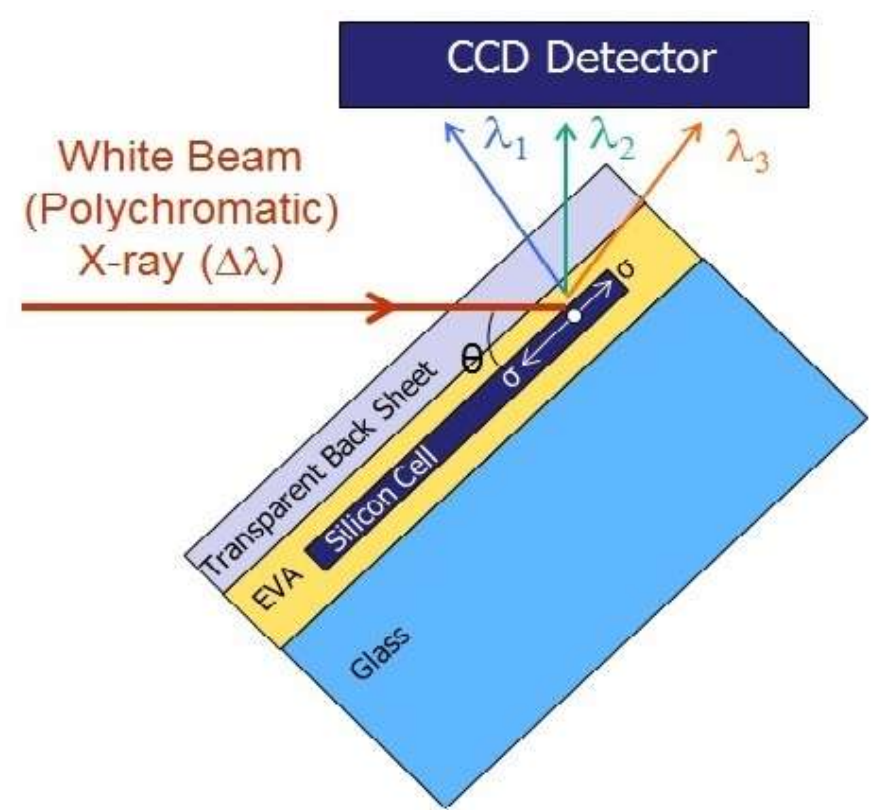

Figure 2: (a) The experimental setup using $\mu$ SXRD, where the mini module mounted on a XY piezoelectric sample stage tilted at 45\%; (b) The incoming X-ray beam capable of penetrating transparent backsheet and backside encapsulant reaches silicon cell and is later diffracted into the CCD detector [9]

The incoming polychromatic X-ray focused beam striking the PV module sample has diameter approximately $1 \mu \mathrm{m} \mathrm{[22]} \mathrm{and} \mathrm{energy} \mathrm{ranging} \mathrm{from} 6 \mathrm{keV}$ to $22 \mathrm{keV}$. The $\mathrm{X}$-ray is not capable of penetrating the front glass $(\sim 3.2 \mathrm{~mm}$ thick) due to absorption and hence the mini PV module was mounted on the sample stage such that the back metallization of the cell facing the beam as shown in Figure 2. For this reason, only transparent backsheet materials could be used to laminate modules (with Encapsulants A and B) in our experiments. The X-ray beam penetrates through the encapsulation layer ( $\sim 0.5 \mathrm{~mm}$ thick) and back metallization $(\sim 10 \mu \mathrm{m}$ thick) before reaching the underlying crystalline silicon wafer $(\sim 180 \mu$ m thick $)$. 
Referring to penetration plots in Appendix-A, the high energy X-ray beam practically passes though the full thickness of the silicon cell and hence the probed volume is roughly cross sectional area times the thickness of the cell $\left(140 \mu \mathrm{m}^{3}\right)$. However since the X-ray beam is polychromatic, penetration depth is a strongly dependent on X-ray energy and consequently it can be concluded that our measurement is volumetric although signals from the outer surface of the cell will have much stronger influence. Furthermore, the through thickness stress gradients, which are expected to be smaller as the silicon cell is a thin wafer are neglected. In addition, it is clear that the encapsulant (EVA) is completely transparent to X-ray beam. While the back metallization does not completely block the X-ray beam (please refer to Appendix-A), the intensity of underlying silicon diffraction signal drops considerably compared to that of bare silicon without metallization. In case of solder joint / interconnect, as the thickness of the solder joint / interconnect is much higher, there will be no penetration and hence no diffraction signal from underlying silicon was detected.

The sample stage was adjusted properly in $x-, y$ - and z- directions to ensure optimal beam focus $(\sim 1 \mu \mathrm{m})$ on the sample using customized laser triangulation system [22]. The beam focus is in general insensitive to small variations $(\sim 10 \mu \mathrm{m})$ in the thickness of the silicon cell as the X-ray penetrates though the thickness of the cell. Subsequently, automatic pixel-by-pixel raster scan was performed on the selected region of the sample (Figure 1a) within the sample yielding Laue diffraction pattern for each pixel. The spacing between pixels was chosen to be $0.1 \mathrm{~mm}$ under a 1.5 second beam exposure to ensure reasonable brightness of the diffraction pattern. Figure 3 shows the typical Laue diffraction pattern for silicon being indexed manually using XMAS software [25] to identify the miller indices of the planes that produce the diffraction spots within the pattern. This indexed pattern can then be used to index the remaining diffraction patterns automatically by XMAS. Once all diffraction patterns are fully indexed, the information about grain crystal orientation/deformation, deviatoric stress/strain tensors, subgrain structures and cell plane orientation angles can be evaluated quantitatively.

Recall that the presence of back metallization reduces the intensity of the diffracted 
signal from the underlying silicon cell. The XMAS software was equipped with a specialized algorithm called "Adaptive Indexing" [25] to overcome such circumstances. Weak silicon diffraction peaks can be detected and indexed by the adaptive indexing by digitally removing all the peaks indexed as copper and from the remaining, XMAS will index as silicon peaks. The experimental setup in the beamline 12.3.2 provides the capabilities to obtain both deviatoric and hydrostatic stress components [25-28]. The following sections will discuss both the residual stress measurement from the white beam Laue diffraction in detail.

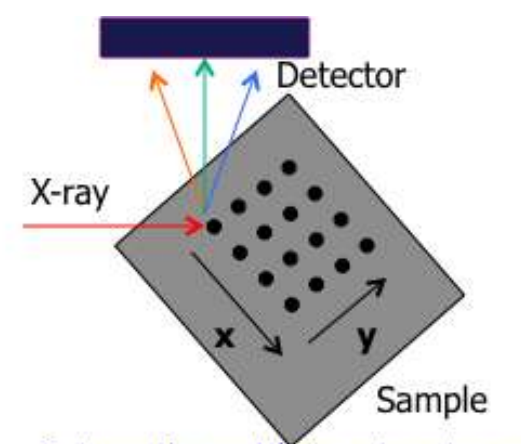

Automatic spot by spot raster scan

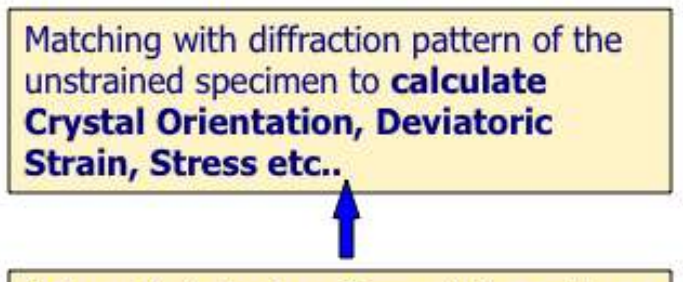

Automatic indexing of remaining patterns

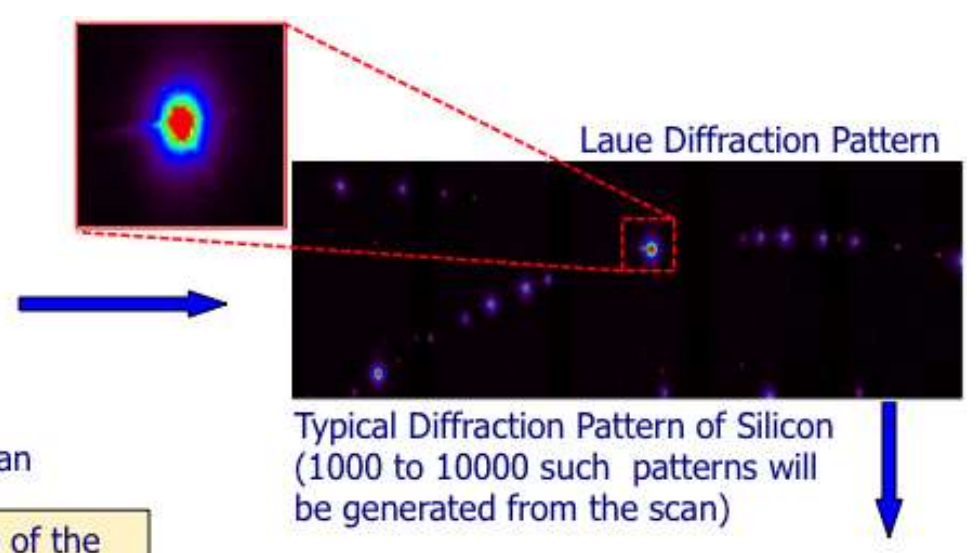
(1000 to 10000 such patterns will

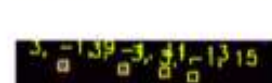

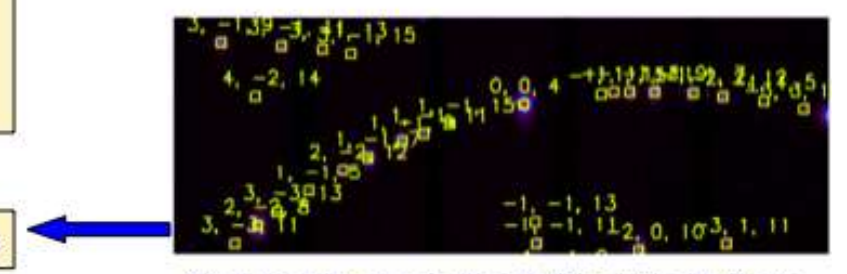

Indexed Peaks in the Diffraction Pattern

Figure 3: Mapping process of X-Ray microdiffraction and subsequent analysis using XMAS [25]

\subsection{Residual deviatoric stress evaluation from the white beam Laue diffraction}

Though Bragg's law is the underlying principle for the standard X-ray diffraction, the specific case of single crystal sample subjected to the white X-ray beam follows Laue diffraction condition, named after Von Laue. The resulting diffraction pattern is commonly known as Laue diagram / pattern (Figure 3) and each spot inside is called as Laue spot. Each Laue spot is generated by a specific X-ray energy selected by a given lattice plane. 
Recall the specialized software program, XMAS [25], which can be used to analyze these patterns and index them as shown in Figure 3, identifying individual patterns from each grain. From this analysis, it is possible to determine the crystal orientation of each grain. As our sample is single crystal silicon, there is only one grain. Furthermore, the deviatoric strain can be derived from the Laue diffraction pattern based on the homogeneity property stating that the coordinates of a given point inside the un-deformed unit cell remain unaffected in the deformed cell as shown below in the equation 1 [25]:

$$
R_{0} X_{0}=R X
$$

$\boldsymbol{X}_{\boldsymbol{O}}$ and $\boldsymbol{X}$ are the coordinate positions of a point, expressed in the Cartesian coordinate system, attached to the unit cell in the undeformed and deformed unit cells respectively whereas $\boldsymbol{R}_{\boldsymbol{o}}$ and $\boldsymbol{R}$ are the transformation matrices converting Cartesian coordinates into unit cell coordinates for the undeformed and deformed unit cell respectively. Equation 1 can be rewritten to define the deformation matrix, T, mapping the undeformed state to the deformed state as shown below:

$$
X=R^{-1} R_{0} X_{0}=T X_{0}
$$

Now the deviatoric strain can be calculated by the equation 3 as given below:

$$
\epsilon_{i j}^{\prime}=\frac{1}{2}\left(T_{i j}+T_{j i}\right)-\delta_{i j}
$$

where $\delta_{i j}$ is the Dirac-delta function. The deviatoric stress is then found using Hooke's law as shown in Equation 4:

$$
\sigma_{i j}^{\prime}=C_{i j k l} \epsilon_{k l}^{\prime}
$$

Further references $[25,29$, and 30] discuss the stress derivation more elaborately.

\subsection{Residual normal (hydrostatic) stress evaluation}

The actual hydrostatic stress measurements of the laminated silicon solar cells here would need a monochromatic beam experiment for each pixel corresponding Laue scan that typically is manual and thus very time consuming. The total normal stress is the sum of 
the normal deviatoric and hydrostatic stress components as shown in Equations 5 and 6. However, the magnitude of normal deviatoric stress component are lower and hence, the hydrostatic stress typically represents the bulk of the total normal stress which will be the subject of this section. The notation of the stresses below is based on the standard definition as described in the literatures $[12,29]$.

$$
\begin{gathered}
\sigma_{i j}=\sigma_{i j}^{\prime}+\sigma_{H} \delta_{i j} \\
\sigma_{H}=\frac{\sigma_{11}+\sigma_{22}+\sigma_{33}}{3}
\end{gathered}
$$

$\sigma_{H}$ is the hydrostatic stress. To produce the typical maps of the residual deviatoric stresses such as shown later in this manuscript (Figures 5 - 10) for the corresponding hydrostatic stress using monochromatic method would require prohibitively much longer time and thus impractical in synchrotron circumstances. To circumvent this problem, we adapted to an indirect method of stress approximation using the crystal plane misorientation angles of the silicon cell wafer $(\Theta)$, as shown in Figure $4 \mathrm{a}$, as a measure of curvature. This approach has an advantage, in addition to being much faster in acquiring the data, the crystal plane misorientation angle of the silicon cell wafer $(\Theta)$ will be negligibly affected by the penetration of the X-ray beam across the sample unlike the stress (or strain), due to the uniformity of the diffraction volume across the scan. Hence, any error resulting from the distortion of the diffraction signal will remain constant over the scan and cancel out during the misorientation angle of the silicon cell wafer $(\Theta)$ calculation.
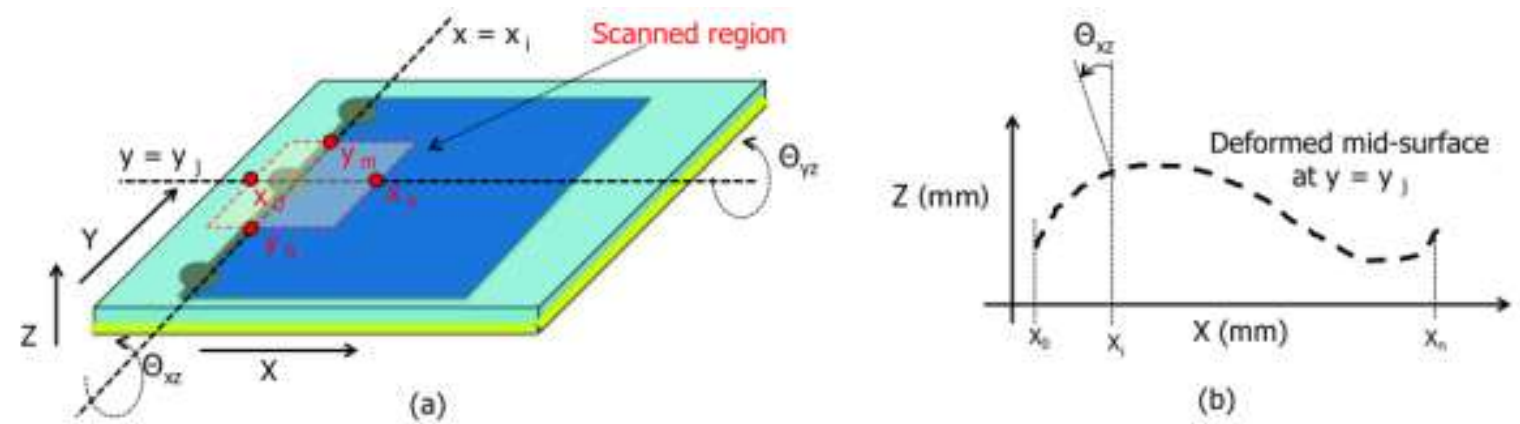

(b)

Figure 4: (a) Schematic of solar PV laminate, showing $\mu$ SXRD scan region, its orientation and associated crystal plane misorientation angles in XZ plane, $\Theta_{\mathrm{xz}}$ and YZ plane, $\Theta_{\mathrm{yz}}$. (b) Schematic of local curvature calculation using equation (7). 
As a result, the curvature of the unit cell at any direction can be calculated by differentiating $\Theta$ with respect to the spatial coordinate. For example, the cell misorientation angle about $y$-axis (in $X Z$ plane), $\Theta_{x z}$ can be obtained as shown in Figure 4, and then Equation 7 can be used to calculate the local curvature of the cell inducing stress in X-direction (please see Figure 4a). Similarly curvature causing Y-direction stress can also be calculated from crystal plane misorientation angle, $\Theta_{y z}$. Since the silicon wafer is very thin compared to its planar dimensions, stress in thickness direction (Z-direction) can be neglected.

$$
\kappa_{x x}=\left.\frac{d \Theta_{x z}}{d x}\right|_{y=c o n s t}
$$

Further upon calculating the curvatures, the respective bending strains and stresses in the cell can be approximately calculated using thin plate theory. Once the directional stresses are known, hydrostatic stress can be approximated using Equation 6.

We observed that the absolute values of cell misorientation angles around the solder joint tend to increase with the increase of distance from the solder joint, monotonically. This curvature technique, thus in our specialized geometry of the sample (with the solder joint), could therefore be simply reduced to the comparison between the maximum misorientation angles either in the $X Z\left(\Theta_{x z}\right)$ or $Y Z\left(\Theta_{y z}\right)$ planes following our earlier observations as reported in $[8,9,33]$. The maximum bending stress in the silicon solar cell wafer as observed in the present study indeed follows the bending pattern as observed in our earlier studies $[8,9,33]$ in the presence of the given solder joint. The maximum stress occurs right at the solder joint edge and decays with the increasing distance from the solder joint. As the solder joint location is nominally standardized in the silicon solar cells (the solder joint process here is a high volume, highly automated manufacturing process), its position thus is nominally at a constant magnitude from the edges of the silicon solar cells. The maximum misorientation angles can simply be compared consequently. The higher misorientation $\left(\Theta_{x z}\right.$ or $\left.\Theta_{y z}\right)$ would be strongly correlated with the bending stress (in $x$ or $y$-direction respectively) in the silicon solar cells. 
While this technique is indeed an indirect approximation, we adopt this approach for the present manuscript to provide fresh insights into the rather pressing questions of the effects of encapsulations on stress state and fracture propensity of silicon solar cells. Some encapsulants may be preferred in the PV industry for higher performance/photon absorption, but could lead to higher cell crack rates in the laminated systems. Thus, such a fast evaluation method would prove to be useful and crucial in enabling further innovation in the next generation PV systems. In the meantime, the authors have indeed ongoing investigations as well to provide the direct measurements of the hydrostatic stress state

following the direct measurement methodologies such as detailed in references [25-30] further for the same samples in the present study.

\section{$3 \quad$ Results and Discussion}

The following sections present the experimental data, as obtained from the Synchrotron X-ray Microdiffraction ( $\mu$ SXRD) experiments using both the above methodologies, which is eventually correlated with the residual stress state within the silicon solar cells laminated by two different polymer materials, Encapsulants A and B, as defined in the Experimental Setup section earlier in this manuscript.

\subsection{Residual deviatoric stress measurement}

The complete stress state (all six components of the second degree tensor) is important in the study of deformation mechanisms in general in any complex advanced device/system. The normal stresses usually lead to fracture events while the deviatoric components could potentially lead to many other mechanical damages such as plastic deformation, interfacial delamination and surface damages. Figures 5 - 10 here show maps of different components of the deviatoric stresses in the silicon solar cell samples laminated by Encapsulants A and B as measured using the methodology described above in Section 2.2. The location of the scanning is the same for both samples with Encapsulants A and B as shown in Figure 1(a) earlier in the manuscript, i.e. around the solder joint (which 
is nominally processed for both samples). However, due to different circumstances during the beamtimes for these two samples, the maps for the two samples differ a little as shown below. Sample with Encapsulant A was scanned with white-beam synchrotron XRD in the area approximately $18 \mathrm{~mm}$ in length ( $x$-direction in the figures) and $10 \mathrm{~mm}$ in width $(y$ direction in the figures), while Sample with Encapsulant B was scanned in the area of approximately $14 \mathrm{~mm} \times 11 \mathrm{~mm}$. As a result, the maps in the figures 5-10 below are shown to scale. However, all these maps evidently showed sufficient information of the stresses around the solder joint (i.e. at least a few millimeters from the solder joint that is the stress concentration point).

$\mathbf{a}$

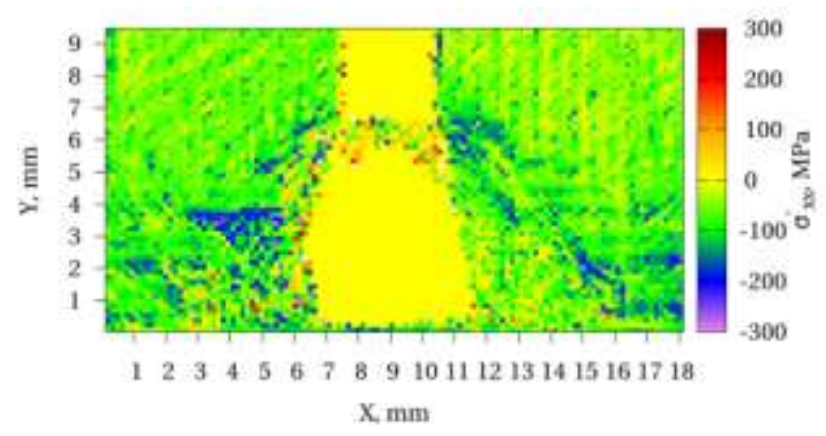

b

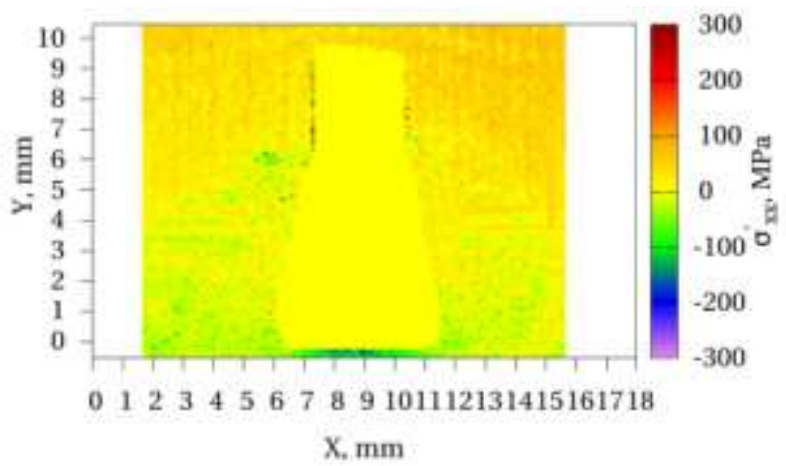

Figure 5: Deviatoric stress in the $x$ direction $\left(\sigma_{x x}^{\prime}\right)$ in the silicon solar cells (around the solder joint) laminated by (a) Encapsulant A, and (b) Encapsulant B.

a

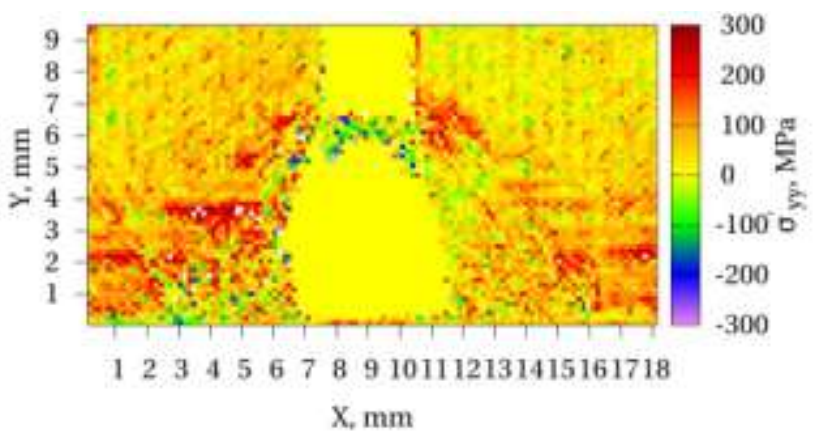

$\mathrm{b}$

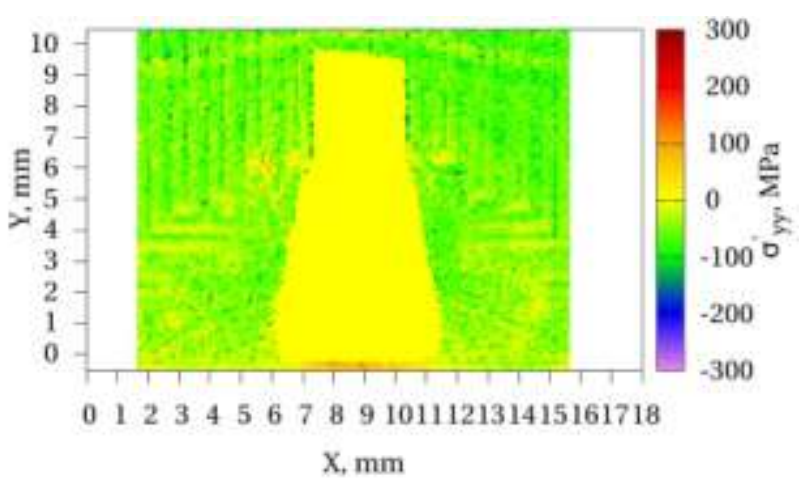

Figure 6: Deviatoric stress in the $y$ direction $\left(\sigma_{y y}^{\prime}\right)$ in the silicon solar cells (around the solder joint) laminated by (a) Encapsulant A, and (b) Encapsulant B. 


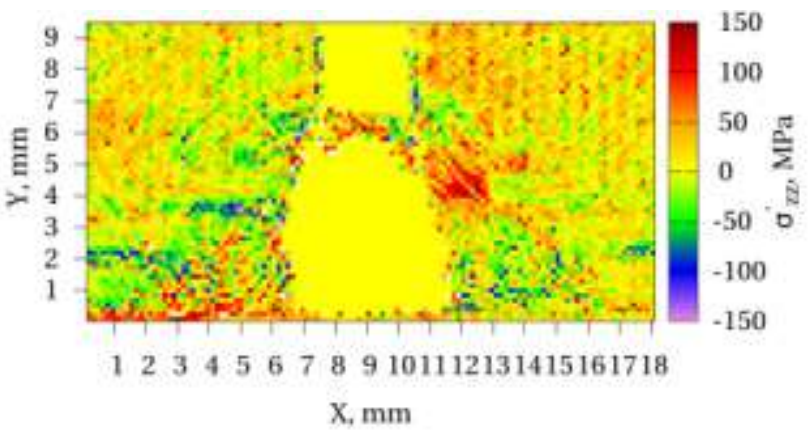

$\mathrm{b}$

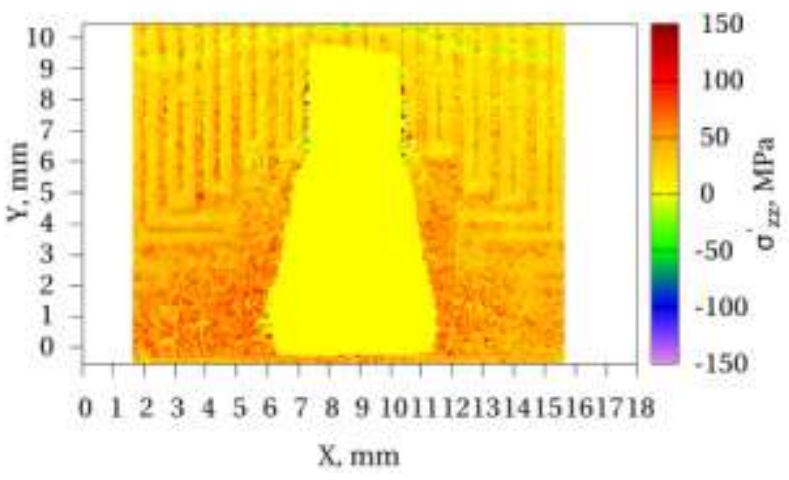

Figure 7: Deviatoric stress in the $z$ direction $\left(\sigma_{z z}^{\prime}\right)$ in the silicon solar cells (around the solder joint) laminated by (a) Encapsulant A, and (b) Encapsulant B.

a



123456789101112131415161718

$\mathrm{X}, \mathrm{mm}$

$\mathrm{b}$



Figure 8: Deviatoric stress in the $x y$ plane $\left(\sigma_{x y}^{\prime}\right)$ in the silicon solar cells (around the solder joint) laminated by (a) Encapsulant A, and (b) Encapsulant B.

a

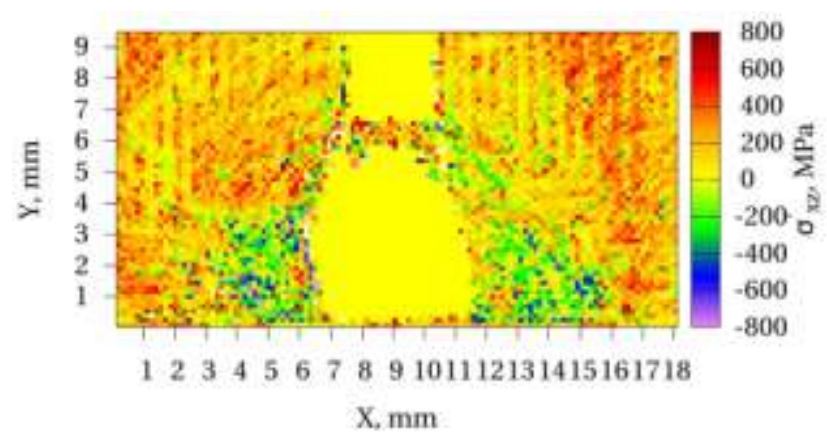

b

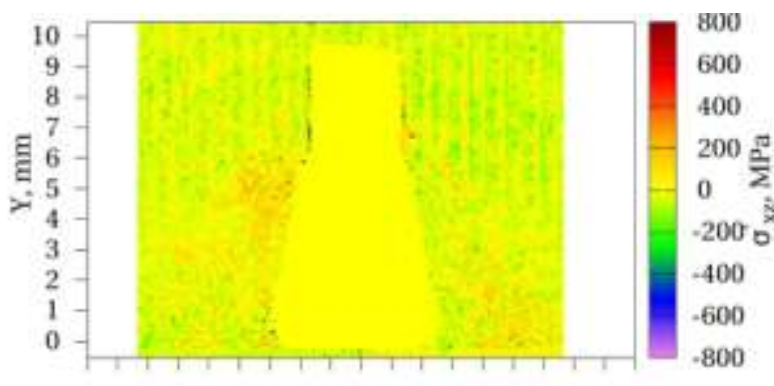

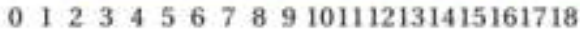

$\mathrm{X}, \mathrm{mm}$

Figure 9: Deviatoric stress in the $x z$ plane $\left(\sigma_{x z}^{\prime}\right)$ in the silicon solar cells (around the solder joint) laminated by (a) Encapsulant A, and (b) Encapsulant B. 




b

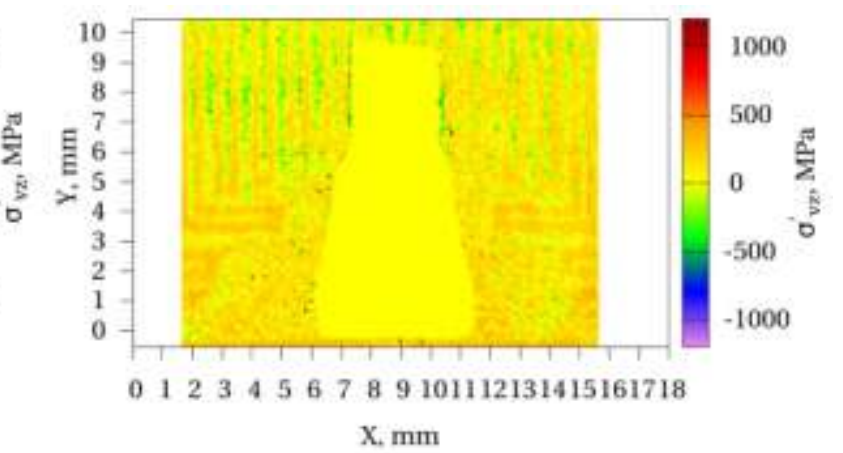

Figure 10: Deviatoric stress in the $y z$ plane $\left(\sigma_{y z}^{\prime}\right)$ in the silicon solar cells (around the solder joint) laminated by (a) Encapsulant A, and (b) Encapsulant B.

a

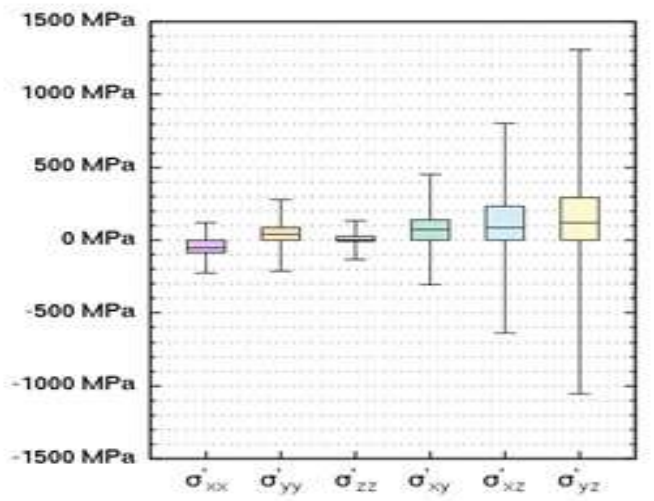

b

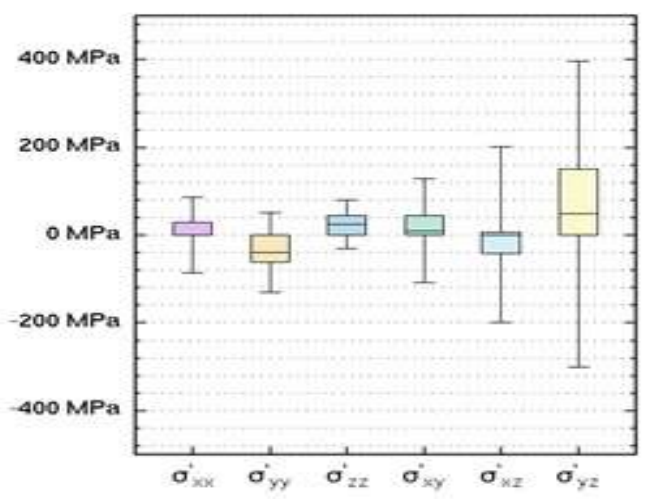

Figure 11: Box plots of all the deviatoric stress components in the silicon solar cells (around the solar joint) shown in Figures 4 - 9 for (a) Encapsulant A, and (b) Encapsulant B.

\begin{tabular}{|c|c|c|}
\hline Deviatoric Stress $(\mathrm{MPa})$ & Sample A & Sample B \\
\hline$\sigma_{x x}^{\prime}$ & 120.5 & 87.2 \\
\hline$\sigma_{y y}^{\prime}$ & 282.5 & 51.9 \\
\hline$\sigma_{z z}^{\prime}$ & 136.3 & 79.5 \\
\hline$\sigma_{x y}^{\prime}$ & 448.7 & 127.8 \\
\hline$\sigma_{x z}^{\prime}$ & 805.5 & 202.4 \\
\hline$\sigma_{y z}^{\prime}$ & 1306.9 & 395.6 \\
\hline
\end{tabular}

Table 1: Summary of maximum values (as defined by the 99\% percentile of all the deviatoric components as shown in Figures 5 - 10 above. Typical deviatoric stress distribution/histogram in similar samples have been shown in our earlier publication [33] 
Figure 11 represents the variation of deviatoric stress magnitudes in all six directions from each solar cells laminated by Encapsulant A and B respectively while Table 1 summarizes the maximum values of deviatoric stresses for each directions within each solar cells. From Figure 11 and Table 1, it is evident that the normal deviatoric stress values in the directions of $x x, y y$ and $z z\left(\sigma_{x x}^{\prime}, \sigma_{y y}^{\prime}\right.$ and $\sigma_{z z}^{\prime}$, respectively) are not very significant compared to the shear stresses $\left(\sigma_{x y}^{\prime}, \sigma_{x z}^{\prime}\right.$ and $\left.\sigma_{y z}^{\prime}\right)$. This could be because much of the stresses in these directions belong to the hydrostatic stress, which is not captured by the Laue/white beam technique (the hydrostatic component will be discussed in the following section). There is a difference here between the stresses in the silicon cells laminated by Encapsulants $\mathrm{A}$ and $\mathrm{B}$ of about one to two hundreds of $\mathrm{MPa}$, but this difference might prove to be not very significant compared to the potential difference in the hydrostatic components.

Further localized shear stresses with very high magnitudes were observed (Figures 9a and 10a), which can be due to the uncertainties in the diffraction peak positions and geometric model parameters. Due to these errors, the shear stress components (YZ and XZ components) of the deviatoric stress indeed have the possibility of greater uncertainty [34]. Hence, localized high shear stresses were neglected in our discussion of results. However, it is evident from the stress maps that the shear stresses mostly follows the metallization lines on the silicon solar cell, and they are not necessarily due to the solder joint itself. The large values of $\sigma_{x z}^{\prime}$ and $\sigma_{y z}^{\prime}$ are expected to consider the asymmetric bending of the silicon solar cell inducing shear stresses in $\mathbf{X Z}$ and $\boldsymbol{Y Z}$ planes. Interestingly, $\boldsymbol{\sigma}_{x \boldsymbol{y}}$ is also quite significant given that the biaxial stress state with the normal stress in one direction is larger than the other due to the metallization lines that are mostly going in one direction. In the context of silicon solar cell bending and high stress concentration near the solder joint, the shear stresses are probably not a big concern for the silicon material integrity, compared to the large values of the normal stresses. Especially if the normal stresses are tensile as they could lead to cracking and fracture of the solar cells. This problem will be further aggravated if there are micro cracks / defects (carried forward from the wafer cutting process) in the silicon solar cell. Thus, accurate estimation of the normal stresses in the solar cell is of prime importance and the deviatoric stress mapping performed in this 
section is not capable of providing complete information of the normal stresses. The evaluation of the total/hydrostatic normal stress is given in the following section.

\subsection{Residual Normal Stress Evaluation}

Figures 12 and 13 show the crystal plane misorientation angles of the silicon cell wafer $(\Theta)$ of the single crystal silicon cell in both $X Z$ and $Y Z$ planes in the region of the solar cells in the vicinity of the solder joint in Samples A and B (i.e. solar cells laminated by the Encapsulants A and B, respectively) as measured experimentally using the methodology described earlier in the Section 2.3. The location of scanning is again the same for both the samples (i.e. around the solder joint), but the scanning area differs slightly (Sample A was scanned in the area of $18 \times 10 \mathrm{~mm}$, whereas Sample B in the area of $14 \times 11 \mathrm{~mm}$ ) and thus the maps in the Figure 11 are shown to scale. As in Figures 5 - 10, the trace of the solder joint is also evident in these maps (indeed those pixels correlated to the solder joint have no signal which are replaced by zeros for imaging purpose as no diffraction data was actually obtained here for the same reason as explained earlier).

a

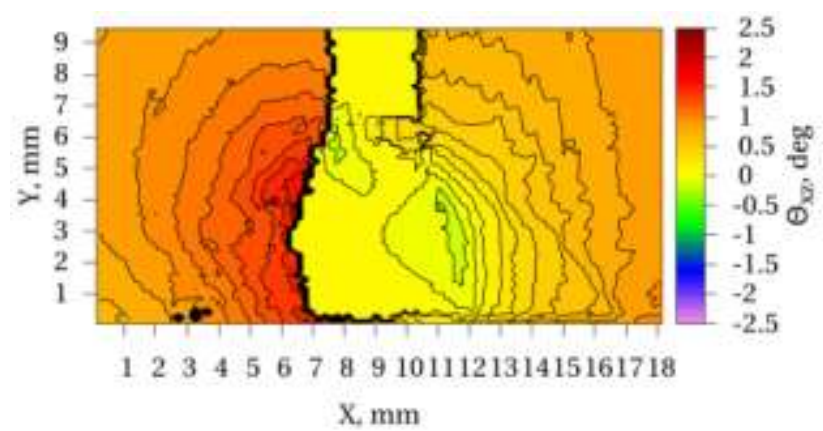

$\mathrm{b}$

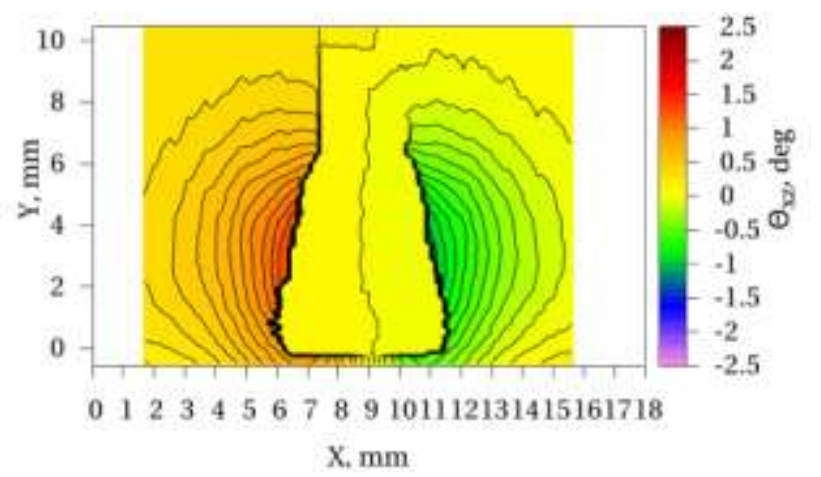

Figure 12: The crystal plane misorientation angles of the silicon cell wafer in $X Z$ plane $\left(\Theta_{\mathrm{xz}}\right)$ in the region of the solar cells in the vicinity of the solder joint in Samples A (a) and Sample B (b). 
a

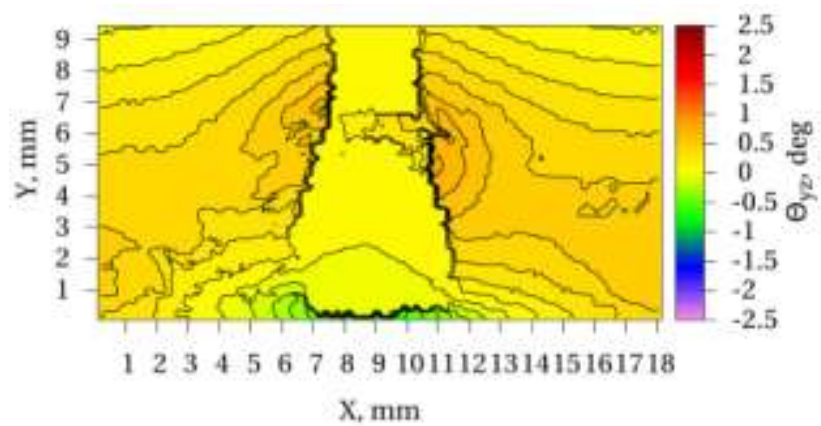

$\mathrm{b}$

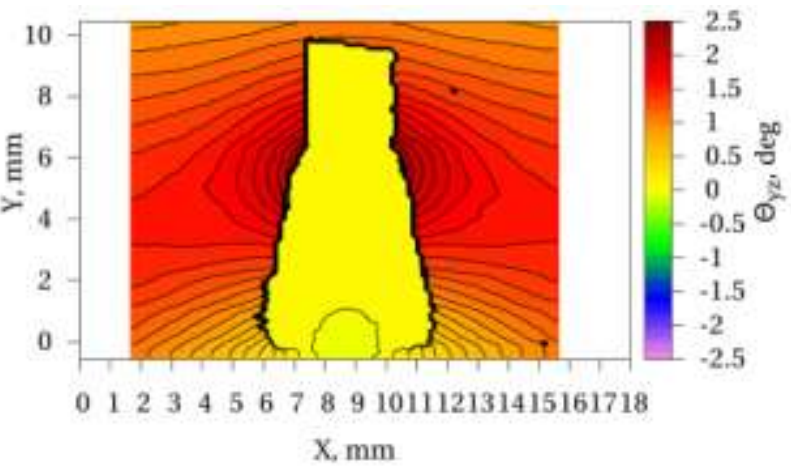

Figure 13: The crystal plane misorientation angles of the silicon cell wafer in $Y Z$ plane $\left(\Theta_{y z}\right)$ in the region of the solar cells in the vicinity of the solder joint in Samples A (a) and Sample B (b).

The crystal plane misorientation angles of the silicon cell wafer $(\Theta)$ in both the $X Z$ and $Y Z$ planes vary significantly on different locations especially on the solder joint edge where there is a significant contrast of misorientation compared to that on the area farther away from the solder joint (the solder joint is indeed a stress concentration point). In this specialized geometry of the sample, as mentioned earlier in the manuscript, the in-plane normal stress comparison (between Samples A and B) could be evaluated in terms of the maximum misorientations, $\Theta$ either in the $X Z$ or $Y Z$ planes, $\left(\Theta_{\mathrm{xz}}\right.$ and $\Theta_{\mathrm{yz}}$, respectively). As the solder joint interconnecting process is a high-volume, highly automated manufacturing process, the position of the solder joint is nominally the same with respect to the silicon solar cell edges. Indeed, for instance, with respect to the $\theta_{\mathrm{yz}}$ data, the maps in Figure 13 show the maximum values to be nominally in approximately the same position (i.e. distance from the bottom edge of the silicon solar cells for both samples). The maximum values of the misorientations, $\Theta$ either in the $X Z$ or $Y Z$ planes, $\left(\Theta_{\mathrm{xz}}\right.$ and $\Theta_{\mathrm{yz}}$ respectively) are summarized in Table 2.

\begin{tabular}{|c|c|c|}
\hline $\begin{array}{c}\text { Maximum } \\
\text { Misorientations }\end{array}$ & Sample A & Sample B \\
\hline$\Theta_{\mathrm{xz}}$ & 1.6401 & 1.3573 \\
\hline$\Theta_{\mathrm{yz}}$ & 0.7360 & 2.2066 \\
\hline
\end{tabular}

Table 2: The maximum values of the misorientations, $\Theta$ in the $X Z$ and $Y Z$ planes 
Table 2 and the maps of Figure 12 and 13 thus show very important observation in terms of the total/hydrostatic normal stress comparison between Samples A vs. B, for two key reasons. First, the fact that all maximum values of $\Theta$, either in the $X Z$ or $Y Z$ planes, $\left(\Theta_{\mathrm{xz}}\right.$ and $\Theta_{y z}$, respectively) are positive means that all total/hydrostatic normal stresses are tensile stresses, which we know are more important as far as the fracture propensity/tendencies and initiation/propagation are concerned. Second, the measured $\Theta_{\mathrm{yz}}$ data of Sample B shows the maximum value of $2.21^{\circ}$, which is significantly higher (close to three times) than the maximum value of the $\Theta_{\mathrm{yz}}$ data of Sample A which is $0.74^{\circ}$. It is also evident that these maximum values occur at the edge of the solder joint at similar distance in $Y$-axis to the bottom edge of the silicon solar cells, which indeed have zero misorientations in terms of $\Theta_{\mathrm{yz}}$ (which should be expected, as the bottom edge of the cell is a free surface/edge and thus, the $\sigma_{y y}$ which is approximated by $\Theta_{\mathrm{yz}}$ should be zero). As the $\Theta_{y z \text { max }}$ in the Sample B is close to three times that in Sample A, it is expected the $\sigma_{y y}$ in Sample B may be approximated to be close to three times that in Sample A. In our earlier report [8], the monochromatic synchrotron X-ray experiments was performed on Sample A (the sample in [8] is indeed the Sample $A$ in the present study), mostly for calibration/verification purposes (not for actual scanning for reasons already explained in this manuscript) and it was obtained that the maximum hydrostatic normal stress of Sample A is in the range of $300 \mathrm{MPa}$ (which is found to be in good agreement with the maximum $\sigma_{y y}$ value essentially obtained through the curvature method [8] which has also been described in Section 2.3 in this manuscript). Based on this earlier finding, it can be estimated that the maximum $\sigma_{y y}$ value in Sample B may well be in the range of $900 \mathrm{MPa}$ (i.e. approximated to be close to three times the maximum $\sigma_{y y}$ value in Sample A).

This is significant as these tensile normal stresses in Sample B are now well beyond the range of typical fracture strengths of silicon in the forms such as used in these samples (i.e. silicon wafers cut from ingot to thickness of $150-250 \mu \mathrm{m}$; the fracture strengths are in the range of $1 \mathrm{GPa}[36,37])$. These findings also underline the validity of the assumption that was made earlier about the deviatoric normal stresses in our samples. Indeed the observed difference in the maximum $\sigma_{y y}^{\prime}$ in Samples A vs. B in the present study (about 1-2 
hundreds of $\mathrm{MPa}$ ) pales in comparison against the difference in the maximum hydrostatic component of the $\sigma_{y y}$ of the same two samples based on our estimation above (which is about several hundreds of MPa). It is then obvious that the total normal tensile stresses in Sample B are in general higher than in Sample A.

The maximum misorientation in the $X Z$ plane $\left(\theta_{\mathrm{xz}, \max }\right)$, however did not show significant contrast comparing Samples A vs. B, as shown in Figure 12, as well as evident from Table 2. This indicates that the normal hydrostatic stresses in the $X X$ direction are not much different in Samples A vs. B. While non-disclosure agreement with our collaborators who provided samples prevents us from publishing the actual fracture images of the silicon solar cells in the present study, this finding is indeed in good agreement with much of the observations in the literature where many cracks [1-5, 35-36], in similar monocrystalline silicon samples such as the ones used in the present study, initiated from the edge of the solder joint, would propagate at least initially along the $X$-axis (or in the case of samples with ribbons/busbars, equivalently perpendicular to the ribbon longitudinal direction), before later on taking on the weakest paths of the silicon cell crystallographically (usually along the $<111>$ directions of the silicon crystal. This crack propagation thus forms the much known dendritic (or "fern" form involving many crack lines at usually $+/-45^{\circ}$ indicating the $<111>$ crystallographic directions in the silicon cells). Such initial crack propagation is indeed consistent with the one driven primarily by the $\sigma_{y y}$ in the sample setup such as used in the present study (or total normal tensile stress in the longitudinal direction of the ribbon/busbar in the sample setup with ribbons/busbars), and not by the $\sigma_{x x}$ as is approximated by the $\Theta_{y z}$ data.

\subsection{Discussion - The Roles of Encapsulants in the Silicon Solar Cell Stress and Fracture}

From the findings in the present study, it is clear the stress state of the silicon solar cells in the two samples, which otherwise are nominally the same except for the encapsulation materials (Encapsulants A vs. B), are significantly different. In the past few years, the observation of different encapsulants leading to substantial differences in the 
fracture rates of silicon solar cells in the laminated form (i.e. PV modules) have become increasingly obvious and widely accepted [6,7]. Obviously, such different stress states as shown in this present study, would lead to very different silicon cell crack rates, and fracture mechanisms in general between Samples A vs. B. In terms of the normal (total/hydrostatic) tensile stresses, which are more critical as far as fracture is concerned, Sample B seems to exhibit substantially much higher crack propensity and thus should lead to substantially much higher crack rates. In order to verify such expected effects on fracture rates, we conducted the in-laminate three-point bending/fracture tests. In this test, two groups of samples, each containing $10 \mathrm{PV}$ mini-modules (one group consisted samples laminated by Encapsulant A and the other by Encapsulant B) were prepared. The PV mini module samples were placed on two parallel supporting tabs with a certain distance and then a uniform quasi static loading was monotonically applied in the center of the upper side of the PV mini-module (i.e. the glass side) by means of a loading tab. As the load increased monotonically, visual inspection was conducted via a camera transmitting the magnified images to a computer and allowing visual/manual detection of macroscale cracks in the silicon solar cells. At the first sight of macroscale cracks in the silicon solar cells, the loading force was recorded and indicated as "Characteristic Strength" which is an indication of the sample resistance to macroscale cracks. Details of this test have been reported elsewhere [9].

The characteristics of fracture load on both Samples A vs. B (i.e. PV mini-module laminated by Encapsulants A vs. B) as obtained through the in-laminate three-point bending experiment are shown in Figure 14. It indicates that fracture load of $1.2 \mathrm{kN}$ was approximately required to induce crack formation on silicon cells laminated by Encapsulant A. This fracture load value drops significantly into approximately $0.3 \mathrm{kN}$ to induce crack formation on silicon cells laminated by Encapsulant B. Despite some statistical variations within each group of the samples, the fracture load results between the two groups of samples are significantly different. Therefore, PV mini-modules with Encapsulant A did seem to resist significantly higher external loads before macroscale crack propagation became visible. This fracture tests are thus in good agreement with the synchrotron X-ray stress findings in the present study. Sample A in the synchrotron study which has much lower normal (total/hydrostatic) tensile stress compared to Sample B, did 
indeed correlate with the PV mini-modules having much higher resistance towards macroscale crack propagation in the silicon cells as shown in Figure 14.

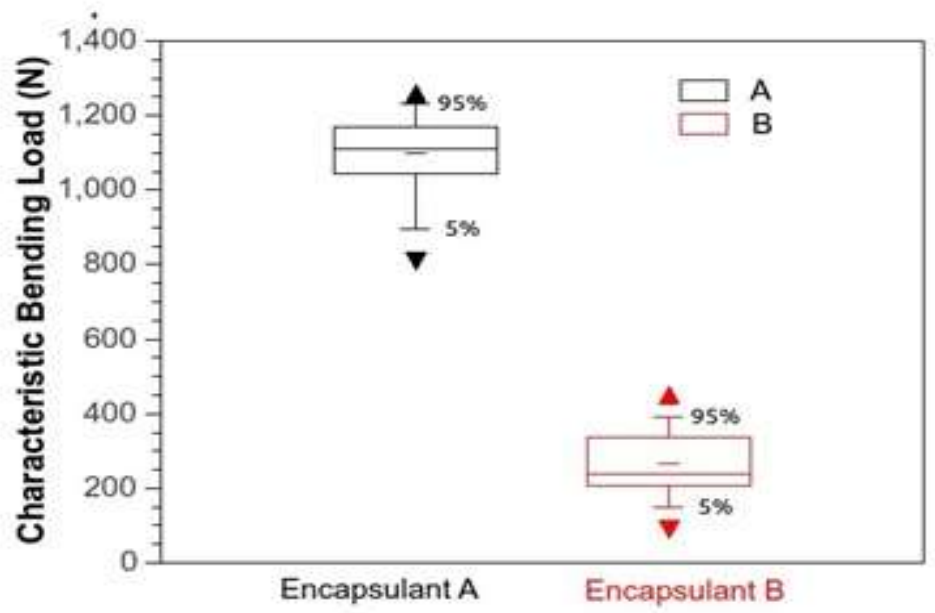

Figure 14: Characteristic fracture strengths of Samples A vs. B (i.e. PV mini-module laminated by Encapsulants A vs. B). [9]

Furthermore, in order to understand the underlying fundamental mechanism responsible for inducing residual stress during the lamination process, the authors also studied the mechanical properties of the two encapsulant materials that we used in the present study (i.e. Encapsulants A and B), through Dynamic Mechanical Analysis (DMA) experiments. Figure 15 compares the storage modulus for both Encapsulants A and B as obtained from the DMA experiment. The result shows that at temperatures ranging from $20-100^{\circ} \mathrm{C}$, Encapsulant $\mathrm{B}$ has higher storage modulus approximately by two orders of magnitudes than that of Encapsulant A. Thus, the DMA experiment confirms that the Encapsulant B is indeed stiffer than Encapsulant A. We believe this is also including at high temperatures typically experienced by the PV mini-module during the lamination process. The DMA data is indeed only valid until $100{ }^{\circ} \mathrm{C}$ only, however, looking at the monotonous and gradual nature of the storage modulus as a function of temperature (as shown in Figure 15), it can be concluded that the Encapsulant B would be stiffer than Encapsulant A even above $100{ }^{\circ} \mathrm{C}$. Consequently, during cooling process from lamination temperature to room temperature, the Encapsulant B is expected to transfer more warpage load from glass to cell creating higher stress in the cell than that that from Encapsulant A. Also perhaps 
quite importantly, during lamination process, the module being fabricated was subjected to a vacuum pressure $(\sim 0.1 \mathrm{MPa})$ in order to remove the air trapped at the interfaces of different layers (glass, encapsulant, cell). Because of that, the softened solder bump (at lamination temperature of $\sim 150{ }^{\circ} \mathrm{C}$ ) around the interconnect/busbar would get distorted and spread around. The level of distortion and spreading is clearly a function of encapsulant stiffness and viscosity. We observed this in the samples made of Encapsulant A (EVA) and not so much with Encapsulant B. This observation is again correlating well with our DMA conclusion that the Encapsulant B is stiffer than Encapsulant A.

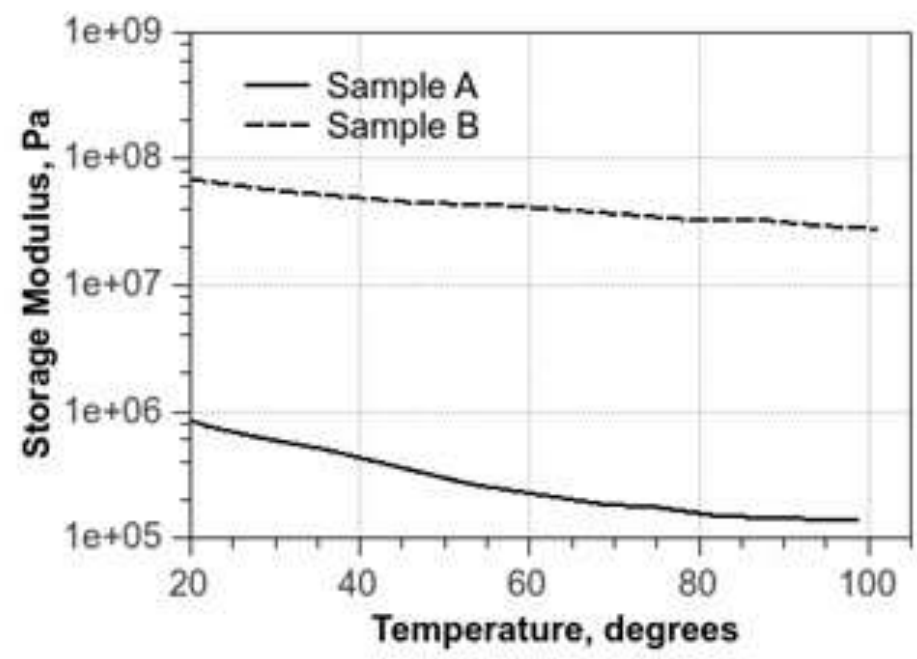

Figure 15: Dynamical Mechanical Analysis (DMA) experiment of Encapsulants A and B [9]

In summary, stiffer encapsulant (i.e. Encapsulant B) transfers more warpage (during lamination) and external load (upon mechanical testing / operation) on solar cells. These observations are consistent with the fracture data characteristics that show Samples A i.e. with Encapsulant A) could delay much of the crack initiation and propagation in the silicon cell up to $1.2 \mathrm{kN}$ of bending force, while Samples B (i.e. with the stiffer Encapsulant B) was already cracking at much lower bending force of about $0.3 \mathrm{kN}$. The increase or decrease of normal tensile stress concentration due to different encapsulation materials may be solely attributed to encapsulant stiffness or may be due to local change of solder joint topology under lamination temperature and pressure in the presence of a very compliant encapsulation material. The exact mechanisms predicting the role of encapsulation material leading to the substantial difference in residual stress and fracture rates observed 
in this study are a subject of our continued investigations with the use of Synchrotron X-ray Microdiffraction. Further in this manuscript, the authors only focused on the effect of encapsulation material on the residual stress in silicon cells with identical soldered condition i.e., nominally same post soldering stress. However, our currently ongoing investigation is aimed to answer the obvious question of residual stress evolution during PV module integration process, from soldering to encapsulation using both Synchrotron Xray Microdiffraction experiments and computational simulations.

\section{Conclusions}

Mechanical stress and microcracks are the origins of failures of the silicon solar PV during fabrication and field operations. Thus, it is very important to have the tools to characterize them in a more quantitative manner. Through the studies in this article, Synchrotron X-ray Microdiffraction ( $\mu$ SXRD) was used for residual stress evaluation in encapsulated solar cells and it proved to be a very useful technique with unique capabilities to characterize stress quantitatively especially once the solar cells are laminated. Further experiments such as in-situ and in-operando evaluation of the silicon solar cell modules are thus enabled using this technique to get the complete understanding of the stress evolution leading to the catastrophic, macroscale fracture events in the silicon solar cells inside the modules. The present study has shown the stress states of silicon solar cells as the result of lamination using different polymers/encapsulants. While the exact mechanisms leading to these findings as well as to the known fracture rate difference are a subject of our continued investigations, this technique has now opened possibilities for us to understand the complete evolution of the mechanical stress and how it may thus lead to lower stress and subsequently lower fracture rates in the silicon solar cells. With such low-stress and low-cracking rate, encapsulation technology could lead to next generation of lower cost silicon solar PV systems. 


\section{Acknowledgements}

The authors would like to thank for critical discussion with Solar Energy Research Institute (Singapore), SunPower Corporation (USA) and REC Solar (Singapore). The authors gratefully acknowledge the discussion and support provided by Dr. Alexander Caldwell of SunPower for the Synchrotron X-ray Microdiffraction experiments. Critical support and infrastructure provided by Singapore University of Technology and Design (SUTD) and Surya University (Indonesia) during the manuscript preparation is highly appreciated. V. Handara, S. K. Tippabhottla and A.S. Budiman also gratefully acknowledge the funding and support from National Research Foundation (NRF)/Economic Development Board (EDB) of Singapore for the project under EIRP Grant "(NRF2013EWT-EIRP002-017) - Enabling Thin Silicon Technologies for Next Generation, Lower Cost Solar PV Systems" and The Advanced Light Source (ALS) (supported by the Director, Office of Science, Office of Basic Energy Sciences, and Materials Sciences Division, of the U.S. Department of Energy under Contract No. DE-AC02-05CH11231 at Lawrence Berkeley National Laboratory and University of California, Berkeley, California). The move of the micro- diffraction program from ALS beamline 7.3.3 onto to the ALS superbend source 12.3.2 was enabled through the NSF grant \#0416243. 


\section{References}

1. M. Sander, S. Dietrich, M. Pander, M. Ebert, and J. Bagdahn. Systematic Investigation of Cracks in Encapsulated Solar Cells after Mechanical Loading. Solar Energy Materials \& Solar Cells, 111:82-89, 2013

2. A. Gabor, M. Ralli, L. Montminy, S. Alegria, C. Bordonaro, J. Woods and J. Felton. Soldering induced Damage to Thin Si Solar Cells and Detection or Cracked Cells in Modules. 21st European Photovoltaic Solar Energy Conference, 2006.

3. M. Sander, S. Dietrich, M. Pander, S. Schweizer, M. Ebert, and J. Bagdahn. Investigations on Crack Development and Crack Growth in Embedded Solar Cells. Proceedings of SPIE - The International Society for Optical Engineering, 2011

4. M. Köntges, I. Kunze, S. Kajari-Schröder, X. Breitenmoser and B. BjAzrneklett. The Risk of Power Loss in Crystalline Silicon based Photovoltaic Modules due to Micro-Cracks. Solar Energy Materials \& Solar Cells, 95:1131-1137, 2011

5. S. Kajari-Schroder, I. Kunze, U. Eitner and M. Kontges. Spatial and Orientational Distribution of Cracks in Crystalline Photovoltaic Modules generated by Mechanical Load Tests. Solar Energy Materials \& Solar Cells, 95:3054-3059, 2011

6. S. Dietrich, M. Pander, M. Sander, S.H. Schulze and M. Ebert. Mechanical and ThermoMechanical Assessment of Encapsulated Solar Cells by Finite Element Simulation. Proceedings of SPIE - The International Society for Optical Engineering, 2008

7. Sander, M. et al. "Influence of manufacturing processes and subsequent weathering on cell cracks in PV modules", Proc. EU PVSEC 2013, 4AV.5.30

8. A.S. Budiman, G. Illya, V. Handara, W.A. Caldwell, C. Bonelli, M. Kunz, N. Tamura, and D. Verstraeten. Enabling Thin Silicon Technologies for Next Generation c-Si Solar PV Renewable Energy Systems using Synchrotron X-ray Microdiffraction as Stress and Crack Mechanism Probe. Solar Energy Materials and Solar Cells 130, p. 303-308, 2014.

9. K.R. Narayanan, G. Illya, V. Handara, N. Tamura, M. Kunz, and A.S. Budiman. Low Stress Encapsulant? - Influence of Lamination Materials on Stress and Fracture of Thin Silicon Solar Cells as Quantified by Synchrotron X-ray Submicron Diffraction. Proc. Eng. 139, p. 76, 2016.

10. R. Mickiewicz, B. Li, D. Doble, T. Christian, J. Lloyd, A. Stokes, C. Voelker, M. Winter, B. Ketola, A. Norris and N. Shephard. Effect of Encapsulation Modulus on the Response of 
PV Modules to Mechanical Stress. $26^{\text {th }}$ European Photovoltaic Solar Energy Conference, 2011

11. A. Colli, K. Attenkofer, B. Raghothamachar and M. Dudley. Synchrotron X-Ray Topography for Encapsulation Stress/Strain and Crack Detection in Crystalline Silicon Modules, IEEE J. Photovoltaics. 6, p. 1387, 2016.

12. A.S. Budiman, H-A.-S. Shin, B.-J. Kim, S.-H. Hwang, H.-Y. Son, M.-S. Suh, Q.-H. Chung, K.Y. Byun, N. Tamura, M. Kunz, and Y.-C. Joo. Measurements of Stresses in $\mathrm{Cu}$ and Si around Through-Silicon Via by Synchrotron X-Ray Microdiffraction for 3-Dimensional Integrated Circuits. Microelectron. Rel. 52, p. 530-533, 2012.

13. T. Tian, R. Morusupalli, H. Shin, H. Son, K. Byun, Y. Joo, R. Caramto, L. Smith, Y. Shen, M. Kunz, N. Tamura, and A.S. Budiman. On the Mechanical Stresses for Cu ThroughSilicon Via (TSV) Samples Fabricated by SK Hynix, Inc. vs. SEMATECH - Enabling Robust and Reliable 3-D Interconnect/Integrated Circuit (IC) Techology, Proc. Eng. 139, p. 101, 2016.

14. A. S. Budiman, C. S. Hau-Riege, W.C. Baek, C. Lor, A. Huang, H.S. Kim, G. Neubauer, J. Pak, P. R. Besser, and W. D. Nix. Electromigration-Induced Plastic Deformation in $\mathrm{Cu}$ Interconnects: Effects on Current Density Exponent, n, and Implications for EM Reliability Assessment. J. Electron. Mater. 39, iss. 11, p.2483-2488, 2010.

15. C.Y. Khoo, H. Liu, W. Sasangka, R. I Made, N. Tamura, M. Kunz, A.S. Budiman, C.V. Thompson, and C.L. Gan. Impact of Deposition Temperature on the Crystallizations Kinetics of Amorphous GeTe Films. J. Mater. Sci. Soc. 51, p. 1864-72, 2015.

16. A. S. Budiman, K.R. Narayanan, L.A. Berla, N. Li, P. Dickerson, J. Wang, N. Tamura, M. Kunz, W.D. Nix, and A. Misra. Plasticity Evolution in Nanoscale Cu/Nb Single Crystal Multilayers as Revealed by Synchrotron X-Ray Microdiffraction. Mater. Sci. Eng. A, 635, p.6-12, 2015.

17. Y. Kim, A. S. Budiman, J. K. Baldwin, N. Mara, A. Misra, and S. M. Han. Microcompression Study of Al-Nb Nanoscale Multilayers. J. Mater. Res. 27, p. 592-598, 2012.

18. A. S. Budiman, G. Lee, M. J. Burek, D. Jang, S. M. Han, N. Tamura, M. Kunz, J. R. Greer, and T.Y. Tsui. Plasticity of Indium Nanostructures as Revealed by Synchrotron X-Ray Microdiffraction. Mater. Sci. Eng. A 538, p. 89-97, 2012. 
19. A. S. Budiman, S. M. Han, Q. Wei, P. Dickerson, N. Tamura, M. Kunz, and A. Misra. Plasticity in the Nanoscale $\mathrm{Cu} / \mathrm{Nb}$ Single Crystal Multilayers as Revealed by Synchrotron Laue X-Ray Microdiffraction. J. Mater. Res. 27, p. 599-611, 2012.

20. A. S. Budiman, N. Li, J. K. Baldwin, J. Xiong, H. Luo, Q. Wei, N. Tamura, M. Kunz, K. Chen, and A. Misra. Growth and Structural Characterization of $\mathrm{Cu} / \mathrm{Nb}$ Single Crystal Multilayers for in situ Synchrotron Laue X-Ray Microdiffraction Studies. Thin Solid Films 519, p. 4137-4143, 2011.

21. Enabling Thin Silicon Solar Cell Technology, Advanced Light Source (ALS) Highlights, Retrieved June 2013, from http://www-als.lbl.gov/index.php/sciencehighlights/industryals/829-improving-thin-silicon-solar-cell-technology.html

22. Tamura $\mathrm{N}$, et al. a superbend X-ray microdiffraction beamline at the Advance Light Source, eScholarship, University of California Berkeley, USA, 2009

23. A.A. MacDowell, R.S. Celestre, N. Tamura, R. Spolenak, B. Valek, W.L. Brown, J.C. Bravman, H.A. Padmore, B.W. Batterman, and J.R. Patel. Submicron X-ray diffraction. Nuclear Instruments \& Methods In Physics Research Section A-accelerators Spectrometers Detectors and Associated Equipment, 467:Berliner Elektronenspeicherring Gesell Synchrotronstrahlung (BESSY), 2001.

24. N. Tamura, R. S. Celestre, A. A. MacDowell, H. A. Padmore, R. Spolenak, B.C. Valek, N.M. Chang, A. Manceau, and J.R.Patel. Submicron X-ray diffraction and its applications to problems in materials and environmental science. Review of Scientific Instruments, 73:1369-1372, 2002.

25. N. Tamura. XMAS: a Versatile Tool for Analyzing Synchrotron X-Ray Microdiffraction Data. Chapter 4: Strain and Dislocation Gradients from Diffraction. World Scientific, 2014

26. N.Tamura, A.A.MacDowell, R.Spolenak, B.C.Valek, J.C.Bravman, W. L. Brown, R. S. Celestre, H. A. Padmore, B. W. Batterman, and J. R. Patel. Scanning X-ray microdiffraction with submicrometer white beam for strain/stress and orientation mapping in thin films. J. of Synchrotron Radiation, 10:137-143, 2003.

27. N. Tamura, H. A. Padmore, and J. R. Patel. High spatial resolution stress measurements using synchrotron based scanning X-ray microdiffraction with white or 
monochromatic beam. Materials Science and Engineering A-structural Materials Properties Microstructure and Processing, 399:92-98, 2005.

28. N. Tamura, M. Kunz, K. Chen, R. S. Celestre, A. A. MacDowell, and T. Warwick. A superbend X-ray microdiffraction beamline at the advanced light source. Materials Science and Engineering A-structural Materials Properties Microstructure and Processing, 524:28-32, 2009.

29. A.S Budiman. Probing Crystal Plasticity at the Nanoscales, Springer, 2015

30. Gene E. Ice, Judy W.L. Pang. Tutorial on Xray microLaue diffraction, Materials Characterization 60 (2009) 1191 - 1201

31. K. Chen. N. Tamura, W. Tang, M. Kunz, Y.C. Chou, K.N. Tu, and Y.S. Lai. High Precision Thermal Stress Study on Flip Chips by Synchrotron Polychromatic XRay Microdiffraction. J. Appl. Phys. 107 (2010) 063502

32. M.A. Brown et al. A Comparison of Xray Microdiffraction and Coherent Gradient Sensing in Measuring Discontinuous Curvatures in Thin Film: Substrate Systems, J. of Applied Mech., Transactions of the ASME, vol. 73, pp. 723 - 729, Sep 2006

33. S. K. Tippabhotla, I. Radchenko, K.R. Narayanan, G. Illya, V. Handara, M. Kunz, N. Tamura, and A.S. Budiman. Synchrotron X-ray Microdiffraction - Probing Stress State in Encapsulated Thin Silicon Solar Cells, Proc. Eng. 139, p. 123, 2016.

34. A. Poshadel et al., Assessment of Deviatoric Lattice Strain Uncertainty for Polychromatic X-ray Microdiffraction Experiments, J. Synchrotron Rad. (2012). 19, 237-244.

35. M. Köntges, S. Kajari-Schröder, I. Kunze, and U. Jahn. Crack Statistic of Crystalline Silicon Photovoltaic Modules, 26 $6^{\text {th }}$ European Photovoltaic Solar Energy Conf. and Exhibition, p. 3290-3294, 2011.

36. M. Demant, M. Oswald, T. Welschelod, S. Nold, S. Bartsch, S. Schoenfelder, and S. Rein. Micro-cracks in silicon wafers and solar cells: Detection and Rating of Mechanical Strength and Electrical Quality, 29th Solar Energy Conf. and Exhibition, p. 390-396, 2014.

37. C. Yang, F. Mess, K. Skenes, S. Melkote and S. Danyluk. On the residual stress and fracture strength of crystalline silicon wafers, App. Phys. Lett. 102, 021909, 2013. 
38. Dietrich et al. Stress Analysis of Encapsulated Solar Cells by means of Superposition of Thermal and Mechanical Stresses ", Proc. SPIE 8825, Reliability of Photovoltaic Cells, Modules, Components, and Systems VI, 882505 (September 24, 2013)

39. E. Gullikson. Center for X-ray Optics, X-Ray Interactions With Matter, (http://henke.lbl.gov/optical constants/atten2.html).

Appendix- A

In this section, the X-ray penetration depths for different materials used in our PV module samples are plotted. These plots were prepared using the tool provided by Center for X-ray Optics (CXRO) of Lawrence Berkeley National Laboratory [39], considering X-ray beam of $45^{0}$ incidence angle having energy in the range of $5-25 \mathrm{keV}$ (as in the case of our experiments reported in this report). These plots are supplementary information which forms the basis for our claim that the crystal structure, deformation and residual stress/strain in an encapsulated material can be probed non-destructively using Synchrotron X-ray microdiffraction technique.



Figure A1. X-ray beam penetration depth in silicon solar cells [39] 


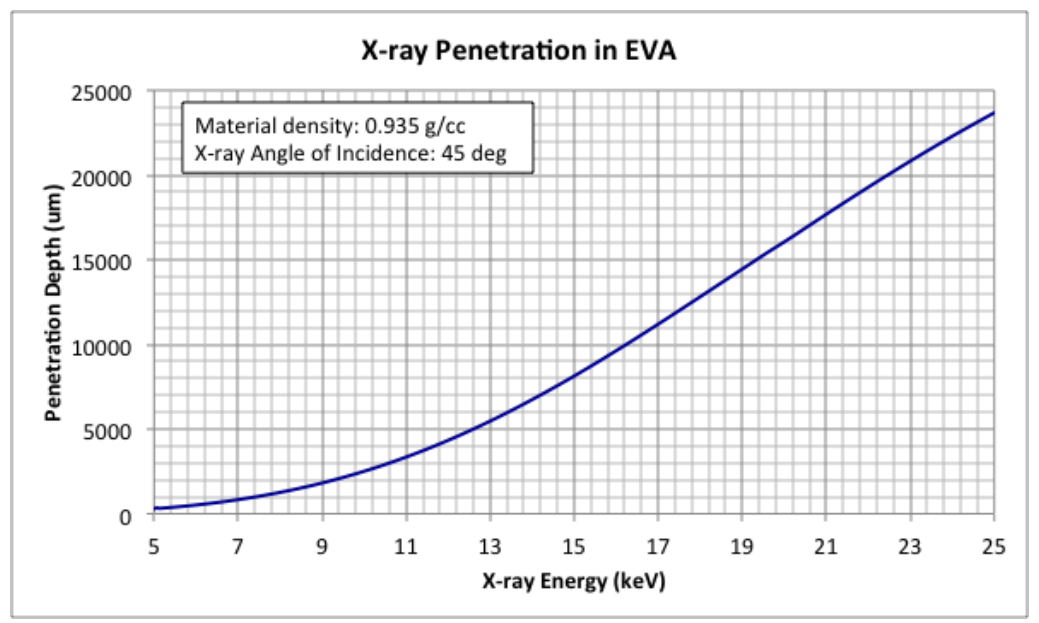

Figure A2: X-ray beam penetration depth in EVA [39]

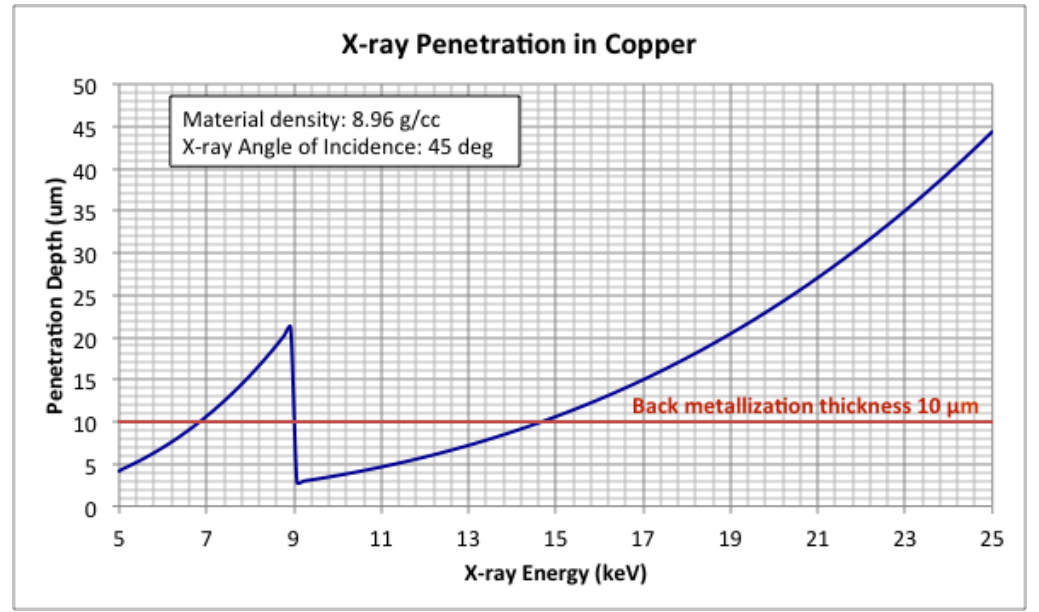

Figure A3: X-ray beam penetration depth in Copper [39]

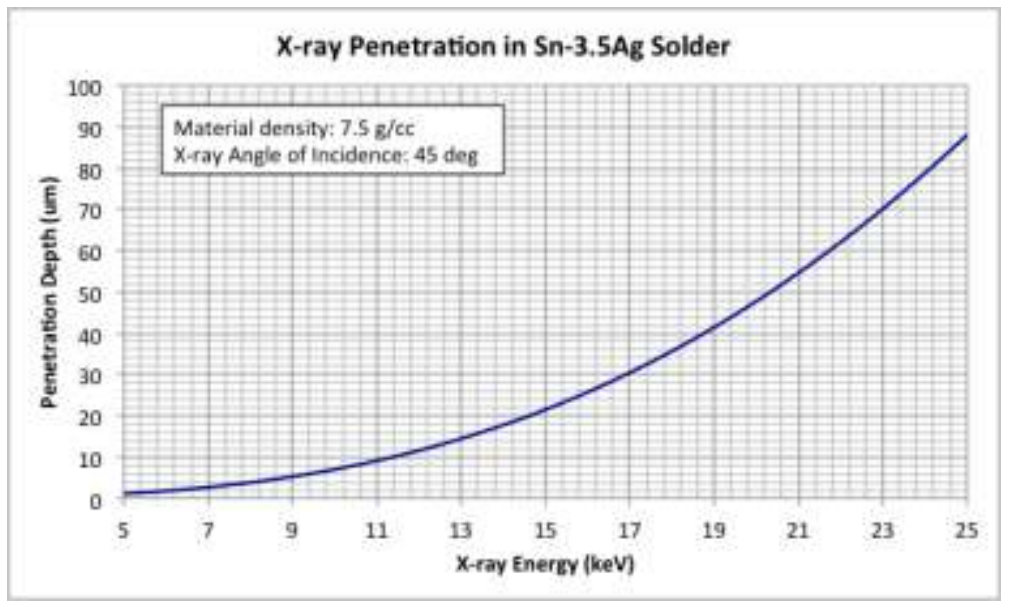

Figure A4: X-ray beam penetration depth in Solder [39] 